\title{
Uma Revisão Teórica da Literatura sobre Empresas Familiares: Posicionamento do Campo Frente às Teorias.
}

\author{
Nome: Bárbara Gabrielle Silva ${ }^{1}$ \\ Centro Federal de Educação Tecnológica de MG \\ e-mail: barbarags96@hotmail.com
}

Nome: Ana Luiz Monteiro Bastos Ornellas ${ }^{2}$

Centro Federal de Educação Tecnológica de MG e-mail: ana_luizamonteiro@hotmail.com

Nome: Uajará Pessoa Araújo ${ }^{3}$

Centro Federal de Educação Tecnológica de MG

e-mail:uajara@cefetmg.br

\section{RESUMO}

A pesquisa sobre empresas familiares vivenciou nos últimos anos mudanças importantes em termos de produção científica. A ascensão da relevância do tema pôde ser percebida com sua crescente apresentação em periódicos reconhecidos e pela criação e consolidação de revistas especializadas na temática. À medida que a literatura expande, encontrar novos horizontes de pesquisa e identificar possibilidades de direções futuras torna-se um importante passo para o avanço do conhecimento. Neste sentido, o presente artigo buscou compreender onde o campo de estudos tem se posicionado atualmente em relação aos temas discutidos e métodos empregados nos últimos anos. Para isso, foi realizada uma revisão da literatura e um levantamento da produção científica publicada em periódicos de reconhecida relevância para a área de empresas familiares. A metodologia empregada foi qualitativa de cunho descritiva e a coleta dos dados realizada através de buscas em bases de dados. Identificaram-se 100 artigos científicos que trataram da temática e as análises foram relacionadas às teorias e metodologias empregadas. Com isso, espera-se que o presente estudo auxilie na abertura de novos caminhos para a pesquisa sobre empresas familiares, contribua para o crescimento do interesse acadêmico e avanço do campo de estudos.

Palavras-chave: Empresa Familiar. Empreendedorismo Familiar. Revisão da Literatura.

\begin{abstract}
Research on family businesses has experienced important changes in terms of scientific production in recent years. The rise of the theme's relevance could be perceived with its increasing presentation in recognized journals and by the creation and consolidation of specialized magazines on the theme. As the literature expands, finding new research horizons and identifying possibilities for future directions becomes an important step towards advancing knowledge. In this sense, the present article sought to understand where the field of studies has currently positioned itself in relation to the topics discussed and methods used in recent years. For this, a literature review and a survey of scientific production published in journals of recognized relevance to the area of family businesses were carried out. The methodology used was qualitative with a descriptive nature and data collection was carried out through searches in databases. 100 scientific articles were identified that dealt with the theme and the analyzes were related to the theories and methodologies used. With this, it is expected that the present study will help to open new paths for research on family businesses, contribute to the growth of academic interest and advancement of the field of study.
\end{abstract}

Keywords: Family Business; Family Entrepreneurship; Literature Review.

[Submetido em 24-jul-2020 - Aceito em: 25-jan-2022 - Publicado em: 25-fev-2022]

${ }^{1}$ Graduação em Administração (CEFET-MG) [(2018)], Mestrado em Administração (CEFET-MG) [(2021)], Doutoranda em Administração University of Central florida [0].

${ }^{2}$ Graduação em Administração (UFV) [(2018)], Mestrado em Administração (CEFET-MG) [(2021)], Doutoranda em Administração (UFLA) [0].

${ }^{3}$ Graduação em Eng. Metalúrgica (UFOP) [0], Mestrado em Administração (UFBA) [0], Doutorado em Eng. de Produção (USP) [0], Doutorado em Administração (LAVRAS) [O]. 


\section{INTRODUÇÃO}

Segundo a Pesquisa Global de Empresas Familiares no Brasil da PWC (2016), as empresas familiares representam $80 \%$ das 19 milhões de companhias que existem no Brasil, contribuindo com aproximadamente 50\% do PIB nacional e constituindo parcela importante do setor corporativo brasileiro. Frente à predominância e representatividade dos negócios familiares e seu significativo impacto econômico, estudos acerca da teoria, pesquisa e prática dessa área vêm se intensificando ao longo dos últimos anos e a pesquisa envolvendo tais organizações vivenciou mudanças importantes em termos de produção científica.

A ascensão da relevância de pesquisas sobre esse tipo particular de arranjo organizacional pode ser comprovada com a crescente apresentação do tema em periódicos reconhecidos e pela criação e consolidação de revistas especializadas na temática. Ao longo dos anos, diversos autores integraram a produção científica sobre o tema abordando diferentes temáticas, (BORGES et al., 2016). Entre os principais pesquisadores que abordaram o campo nos últimos anos, destacam-se Borges e Lima, com publicações relevantes nos anos de 2012 a 2017; Grzybovski, com trabalhos relacionadas a práticas de gestão em empresas familiares; Lescura, Brito, Capelle e Borges com publicações relevantes sobre o tema no ano de 2012 e Oliveira com estudos sobre governança e legitimidade nos anos de 2011 a 2013. Todos com, pelo menos, duas publicações sobre empresas familiares em periódicos de grande relevância nos últimos anos.

Frente à tal realidade, o objetivo da presente pesquisa consistiu em compreender onde o campo de estudos tem se posicionado atualmente em relação aos temas discutidos e métodos empregados nos últimos anos. Para isso, foi realizada um estudo qualitativo de cunho descritivo que, para Gil (2009), tem como principal objetivo descrever as características de determinadas populações ou fenômenos. Como técnica de coleta de dados utilizou-se a revisão sistemática de literatura que para Botelho, Cunha e Macedo (2011) tem como propósito identificar o "estado da arte" do objeto em análise.

A aplicação da técnica de revisão bibliográfica envolveu, em um primeiro momento, o levantamento da produção científica publicada em periódicos de reconhecida relevância para a área de empresas familiares, objetivando compreender como o campo vem se posicionando na literatura acadêmica dos últimos anos. Para se efetuar a busca foram realizadas consultas às bases eletrônicas Web of Science, SciELO (Scientific Electronic Library Online) e SPELL (Scientific Periodicals Electronic Library) com a aplicação dos seguintes filtros: artigos em português e publicados em periódicos de Qualis A1, A2, B1 ou B2 (conforme sistema brasileiro de avaliação de periódicos, mantido pela CAPES - 2019).

Em um segundo momento foi realizada a tabulação dos artigos encontrados e a construção da matriz de dados. Ao final, foram selecionados 100 artigos, cujos conteúdos foram categorizados na matriz segundo os seguintes critérios: (a) Periódico, (b) Qualis, (c) Palavras-Chave, (d) Teoria abordada, (e) Método de pesquisa e (f) técnica de coleta dos dados. Tais critérios foram definidos com base na leitura de alguns metaestudos, como o de Paiva, Oliveira e Melo (2008). Com os dados já descritos na matriz, realizaram-se as análises pretendidas.

Tendo em vista que segundo Borges et al. (2016), a temática sobre empresas 
familiares tem ganhado relevância devido à inserção do tema em periódicos de prestígio e a existência de revistas especializadas, edições especiais, instituições acadêmicas e profissionais engajados em discutir a respeito desse tipo de empresa. Buscou-se neste trabalho reunir recursos para compreender o estado da arte do campo de pesquisa sobre empresas familiares a fim de alcançar o objetivo de compreender onde o campo de estudos tem se posicionado atualmente em relação aos temas discutidos e métodos e técnicas empregados nos últimos anos. Espera-se que a partir dessa revisão, seja possível obter uma ideia precisa sobre o estado atual e as lacunas desse tópico, a fim de alavancar o desenvolvimento do campo científico.

O presente artigo está estruturado em seis partes. A primeira trata-se da introdução na qual apresenta a contextualização e o objetivo deste estudo. A segunda parte refere-se à revisão do campo de pesquisa a respeito das empresas familiares. $\mathrm{Na}$ terceira, quarta e quinta parte os resultados desta pesquisa são apresentados, incialmente os temas identificados são expostos e posteriormente os métodos e técnicas aplicados pela amostra estudada são evidenciados. E, por fim, expõe-se as considerações finais do trabalho.

\section{REVISÃO DO CAMPO DE PESQUISA SOBRE EMPRESAS FAMILIARES}

Para Moraes Filho, Barone e Pinto (2011), ainda não há um consenso na comunidade acadêmica quanto a um conceito que seja capaz definir empresas familiares, visto que cada autor se baseia em diferentes perspectivas e critérios. Tal inconsistência conceitual torna-se um fator restritivo à construção de estudos comparativos acerca de tais organizações e acaba dificultando o aprofundamento das pesquisas em tal campo de estudo.

A expressão "familiar", para Grzybovski e Vieira (2012), deixa implícito que há envolvimento de dois ou mais membros de uma mesma família no negócio, seja como proprietário ou administrador, provocando uma reflexão sobre sua origem e história numa dada conjuntura familiar.

Entre as características que diferenciam empresas familiares de companhias não familiares, estão o poder centralizador e conservador na figura do líder, planos estratégicos em longo prazo com o objetivo de perpetuação dos negócios (planejamento sucessório) e a manutenção do capital estritamente no grupo familiar, (ALMEIDASANTOS et al., 2017),

Leone (2005), por sua vez, argumenta que o conceito de empresa familiar é internacionalmente reconhecido por relacionar três esferas: a) o nível da propriedade (iniciada e controlada por um membro da família); b) o nível da gestão (propriedade e/ou direção ser ocupada pelos membros da família); 3) o nível da sucessão (as próximas gerações assumem o lugar das anteriores). Neste sentido, pode-se inferir que o aspecto familiar de tais organizações se relaciona principalmente ao estilo com que a empresa é administrada.

O presente trabalho adotou a definição de empresas familiares como organizações nas quais os proprietários e gerentes pertencem a uma mesma unidade familiar, exercem influência sobre a gestão organizacional conforme seus interesses e valores, determinam a maior parte das decisões estratégicas, e interferem diretamente no desempenho da empresa, (SHARMA, CHRISMAN, \& CHUA, 1997). 


\section{TEMAS DE PESQUISA IDENTIFICADOS}

Para verificar quais tópicos de estudo vinham sendo discutidos com maior frequência na literatura de empresas familiares, elaborou-se a Tabela 01 abaixo com as principais temáticas identificadas, classificadas em ordem decrescente da mais pesquisada para a menos pesquisada.

Tabela 1. Temas de pesquisas sobre Empresas Familiares

\begin{tabular}{|l|c|c|}
\hline \multicolumn{1}{|c|}{ TEMAS DE PESQUISA } & $\begin{array}{c}\text { TOTAL DE } \\
\text { ARTIGOS }\end{array}$ & $\%$ \\
\hline Processo sucessório & 35 & $35 \%$ \\
\hline Cultura Organizacional & 17 & $17 \%$ \\
\hline Práticas de gestão & 13 & $12 \%$ \\
\hline Governança corporativa & 12 & $5 \%$ \\
\hline Inovação empresarial & 5 & $4 \%$ \\
\hline Gestão de desempenho & 4 & $3 \%$ \\
\hline Empreendedorismo Corporativo & 3 & $2 \%$ \\
\hline Ensaios teóricos & 2 & $2 \%$ \\
\hline Empreendedorismo estratégico & 2 & $2 \%$ \\
\hline Estilos de Liderança & 2 & $2 \%$ \\
\hline Racionalidade & 2 & $2 \%$ \\
\hline Relações Interpessoais & 1 & $1 \%$ \\
\hline Mudança organizacional & 100 & $100 \%$ \\
\hline TOTAL & & \\
\hline
\end{tabular}

Entre os principais temas de pesquisa abordados pelo campo nos últimos anos, o interesse pela temática de Processos Sucessórios possui notório destaque frente aos demais. Trabalhos como o de Pereira et al. (2013), Costa et al. (2015) e Teston e Fillipim (2016) buscam compreender o processo de transição geracional das organizações, enquanto trabalhos como Gonçalves (2000) e Moraes Filho, Barone e Pinto (2011) objetivam interpretar as teorias que dão base a tais processos.

Outros estudos, por sua vez, buscaram estudar o processo sucessório relacionandoo a outras teorias e tópicos de pesquisa. $\mathrm{O}$ maior interesse por pesquisas relacionadas a essa temática se dá, possivelmente, por esta ser a principal característica de uma empresa familiar e, ao mesmo tempo, um de seus maiores desafios conforme pontuam Schneider e Neto (2016).

A segunda temática mais abordada nos artigos selecionados foi Cultura Organizacional que, para Lourenço e Ferreira (2012), muitas vezes, nada mais é do um reflexo dos valores e crenças do fundador. Conceituação essa que se relaciona diretamente com a terceira temática mais abordada -práticas de gestão- em que a maioria dos trabalhos, como Martins et al. (2008), Cançado et al. (2013) e Três et al. 
(2014), buscaram compreender quais aspectos gerenciais diferem uma empresa familiar de outro tipos de negócios, uma vez que nestas empresas, os laços familiares podem criar condições para a obtenção de um desempenho diferenciado em comparação com organizações não familiares.

A quarta temática mais identificada nas pesquisas foi sobre Governança Corporativa, onde estudos como os de Carvalho et al. (2016) e Fonseca et al. (2010) buscaram compreender este processo através de abordagens quantitativas. Já Freitas e Barth (2012) e Bertucci et al. (2009) voltaram seus estudos para a complexidade e os desafios da gestão das empresas familiares, bem como a influência dos elementos da governança corporativa na orientação do processo sucessório em organizações familiares.

Em seguida, as temáticas de Inovação Empresarial e Gestão de desempenho foram as mais presentes nos artigos selecionados, responsáveis por 5\% e 4\% dos temas de pesquisa identificados nas publicações respectivamente. Para Padilla-Meléndez, DieguezSoto e Garrido-Moreno, (2015) a participação da família na gestão de uma organização é capaz de impactar negativamente os recursos disponibilizados para a inovação, porém empresas familiares tendem a realizar resultados positivos quando aplicam técnicas inovadoras. Para os autores, existem inúmeras razões para se estudar a inovação em empresas familiares, embora esse ainda seja um tema incipiente em estudos da área de administração.

Em oitavo lugar estão os estudos relacionados à gestão de desempenho. Sabe-se que com a emergência dos mercados globais e a aceleração contínua das mudanças tecnológicas, as fontes de vantagem competitivas tradicionais já não são mais tão eficazes. Por esta razão, as organizações necessitam cada vez mais de ferramentas que as propiciem uma boa gestão de desempenho, alinhamento, suporte e controle estratégico.

Por fim, os 7 tópicos restantes acomodaram apenas 14\% de todos os artigos pesquisados. Temas como Empreendedorismo Corporativo; Ensaios teóricos; Empreendedorismo estratégico; Estilos de Liderança; Racionalidade; Relações Interpessoais; e Mudança organizacional foram abordados na minoria dos estudos realizados pelos pesquisadores do campo. Além disso, algumas áreas temáticas não foram identificadas em nenhum dos artigos selecionados, como: Casais empreendedores; Processo empreendedor; ou Redes empreendedoras, o que diverge do estudo realizado por Borges et al. (2016) que, ao revisarem 73 artigos publicados sobre empresas familiares, identificaram 9 contendo as temáticas supracitadas.

\section{MÉTODOS DE PESQUISA IDENTIFICADOS}

Além da definição da abordagem de pesquisa a ser utilizada (qualitativa ou quantitativa), para se desenvolver uma pesquisa é indispensável selecionar o método que, de acordo com as características da pesquisa, melhor atenderá as necessidades do(s) pesquisador(es). Neste sentido, poderão ser escolhidas diferentes modalidades, sendo possível até mesmo aliá-las (Gerhardt \& Silveira, 2009).

Desta forma, após a identificação das principais temáticas de estudo nos artigos selecionados, buscou-se verificar quais as abordagens metodológicas e métodos de pesquisa mais presentes nos artigos selecionados. Para tanto, elaborou-se a Tabela 02 abaixo com as principais metodologias identificadas, classificadas em ordem decrescente da mais empregada para a menos empregada. 
Tabela 2. Abordagem e Métodos de pesquisas sobre Empresas Familiares

\begin{tabular}{|l|c|c|}
\hline ABORDAGEM (MÉTODO DE PESQUISA) & $\begin{array}{c}\text { TOTAL DE } \\
\text { ARTIGOS }\end{array}$ & $\%$ \\
\hline Abordagem Qualitativa (Estudo de Caso) & 47 & $47 \%$ \\
\hline Abordagem Qualitativa (Casos Múltiplos) & 28 & $28 \%$ \\
\hline Abordagem Qualitativa (Revisão da Literatura) & 14 & $14 \%$ \\
\hline Abordagem Quantitativa (Casos Múltiplos) & 7 & $7 \%$ \\
\hline Abordagem Quantitativa (Estudo de Caso) & 2 & $2 \%$ \\
\hline Abordagem Qualitativa (Etnografia) & 2 & $2 \%$ \\
\hline TOTAL & 100 & $100 \%$ \\
\hline
\end{tabular}

Com os artigos selecionados foi possível constatar que a abordagem mais empregada em pesquisas sobre empresas familiares é a Qualitativa, identificada em 91\% dos artigos selecionados. A metodologia Quantitativa por sua vez só apareceu em apenas 9\% dos estudos. Nota-se que mesmo apresentando desafios, como maior complexidade durante a coleta e a organização de dados; possibilidade de enviesamento quanto a análise dos dados coletados; e podendo incorrer em questões relacionadas a critérios de validade e fidedignidade (Miles, 1979), a utilização da abordagem qualitativa ainda predomina nos estudos sobre tais organizações.

Após a análise da abordagem, buscou-se verificar quais os métodos de pesquisa mais empregados nos estudos selecionados. Dentro da abordagem Qualitativa o método Estudo de Caso foi o que apresentou o maior número de aplicações, empregado em 47\% do total de artigos selecionados. Esse método, conforme aponta Gil (2009), além de explorar situações reais, também explica determinados fenômenos em situações complexas que certos levantamentos e experimentos não são capazes de fazer, fornecendo o conhecimento aprofundado de uma determinada realidade e permitindo a formulação de hipóteses para o encaminhamento de pesquisas futuras.

Ainda na abordagem Qualitativa, os métodos de casos múltiplos e revisão da literatura foram identificados em $28 \%$ e $14 \%$ dos artigos selecionados, respectivamente. A respeito deste primeiro método, Triviños (1987) argumenta que consiste em estudar dois ou mais sujeitos sem necessariamente compará-los. A revisão da literatura, por sua vez, consiste no processo de busca, análise e descrição de um dado campo do conhecimento em busca de respostas para questionamentos de pesquisa.

A etnografia apareceu em última colocação como método de pesquisa de abordagem Qualitativa, sendo identificada em apenas 2\% dos artigos selecionados. Esse tipo de pesquisa tem como objetivo conhecer determinados estilos de vida e culturas específicas de determinado grupo. Para tanto, consiste na análise, avaliação e descrição de todos os dados possíveis e perceptíveis existentes no contexto pesquisado (Cavedon, 2008).

Entre os métodos de pesquisa empregados nas pesquisas de abordagem Quantitativa estão: casos múltiplos e estudo de caso, com $7 \%$ e $2 \%$ de aplicação, 
respectivamente. O tratamento estatístico no estudo de caso, para Triviños (1987), é mais simples quando a análise é quantitativa. A análise qualitativa, pode ter apoio quantitativo, mas geralmente se omite a análise estatística ou o seu emprego não é sofisticado.

\section{TÉCNICAS DE COLETA DE DADOS IDENTIFICAS}

A escolha da técnica de coleta de dados é fundamental para fortalecer uma determinada investigação e obter dados confiáveis para um estudo. Esta etapa é, portanto, de extrema importância no processo de pesquisa, uma vez que uma análise pode obter resultados totalmente desalinhados com a realidade caso os dados utilizados não sejam passiveis de compreensão ou tenham sido obtidos de maneira imprecisa e sem confiabilidade (Barbosa, 2008).

Não existe uma "melhor técnica de coleta de dados", mas sim, a mais eficaz e eficiente que se aplica à pesquisa a ser realizada. É importante, portanto, estudar bem as técnicas de coleta de dados disponíveis a fim de se realizar uma escolha adequada do método para cada questão de pesquisa. Além disso, elas podem ser aplicadas de maneiras isoladas ou mediante combinação de uma ou mais técnicas (Ferreira, Torrecilha \& Machado, 2012).

Após a identificação dos métodos de pesquisa aplicados nos artigos selecionados, buscou-se verificar quais as técnicas de coleta de dados utilizadas e, para isso, elaborouse a Tabela 03 abaixo com as principais técnicas identificadas, classificadas em ordem decrescente da mais empregada para a menos empregada.

Tabela 3. Técnicas de coleta de dados em pesquisas sobre Empresas Familiares

\begin{tabular}{|l|c|c|}
\hline \multicolumn{1}{|c|}{ TÉCNICA } & $\begin{array}{c}\text { TOTAL DE } \\
\text { ARTIGOS }\end{array}$ & $\%$ \\
\hline Entrevistas semiestruturadas & 59 & $59 \%$ \\
\hline Documental (Base de dados) & 21 & $21 \%$ \\
\hline Entrevistas em profundidade & 9 & $9 \%$ \\
\hline Survey (Questionários) & 7 & $7 \%$ \\
\hline Observação não participante & 3 & $3 \%$ \\
\hline Observação participante & 1 & $1 \%$ \\
\hline TOTAL & 100 & $100 \%$ \\
\hline
\end{tabular}

A entrevista como fonte de coleta de dados tem como objetivo averiguar os fatos, diagnosticando opiniões dos indivíduos e descobrindo possíveis planos de ação para os problemas propostos. A modalidade de entrevista mais aplicada nos artigos selecionados foi a semiestruturada que, segundo Gil (2009), é aquela em que o entrevistador tem liberdade para explorar o assunto com o entrevistado sem um roteiro rígido formalizado podendo se basear apenas pela relação de pontos de interesse previamente estabelecida. Dentre as vantagens da utilização deste método de coleta encontram-se a "oportunidade de obtenção de dados que não se encontram em fontes documentais e são relevantes e significativos" e a "possibilidade de obter informações mais precisas" (Lakatos \& 
Marconi, 2009, p. 83). Possivelmente estas foram algumas das razões pelas quais a entrevista semiestruturada foi a técnica com maior aplicação nos artigos selecionados, presente em $59 \%$ deles.

A segunda técnica mais aplicada nos artigos foi a pesquisa documental em bases de dados (registros institucionais, sites, folders, manuais, documentos das organizações, entre outros). Ainda que esta técnica traga benefícios como a redução no tempo e no custo de pesquisas, também traz certas limitações, como: dados muitas vezes incompletos ou excessivamente agregados, dificultando sua análise; mudanças de padrões e processos ao longo do tempo que inviabilizam a comparação entre dados obtidos em épocas distintas; e a restrição de acesso a dados confidenciais (Barbosa, 2008).

Em seguida, as técnicas mais empregadas foram entrevistas em profundidade $e$ survey, identificadas em $9 \%$ e $7 \%$ dos artigos selecionados. A entrevista em profundidade enquanto fonte de coleta de dados, conforme pontua Moré (2015), não se legitima pela quantidade de participantes e sim pela qualidade, profundidade, detalhamento e contextualização dos relatos coletados. O método survey, por sua vez, é um tipo de investigação quantitativa que possui múltiplas finalidades. Toda vez que o pesquisador estiver interessado em identificar opiniões, atitudes, valores e/ou percepções de uma determinada amostra, ele pode empregar o survey como técnica de coleta de dados. Para isso, o instrumento usualmente aplicado é o questionário estruturado (Paranhos et al., 2013).

As duas técnicas menos identificadas nos artigos selecionados, portanto, foram observação não-participante e observação participante, empregadas em $3 \%$ e $1 \%$ dos artigos, respetivamente. A observação não participante também pode ser conhecida como "simples". Nela, o pesquisador permanece alheio à comunidade a qual está pesquisando, tendo um papel de espectador frente ao objeto observado. Nesta técnica os sujeitos não sabem que estão sendo observados, o observador não está diretamente envolvido na situação analisada e não interage com objeto da observação (Gil, 2006).

Em contrapartida, na observação participante, o pesquisador torna-se parte do contexto observado. Essa prática, segundo Ferreira, Torrecilha e Machado (2012), tem sido utilizada por pesquisadores nos últimos anos para coletar dados sobre as características dos participantes que não são facilmente acessíveis por outros métodos. Essa técnica tem suas origens na etnografia e possibilita ao observador e aos observado desenvolver um relacionamento de confiança, necessário para os participantes revelarem os verdadeiros desafios e realidades de sua vivência.

\section{CONSIDERAÇÕES FINAIS}

O presente estudo buscou compreender onde o campo de estudos tem se posicionado atualmente em relação aos temas discutidos e métodos empregados nos últimos anos. Para alcançar tal objetivo realizou-se uma revisão da literatura publicada em periódicos de reconhecida relevância sobre empresas familiares nos últimos anos.

O presente estudo possibilitou verificar que apenas 4 temas de pesquisa (processo sucessório, cultura organizacional, práticas de gestão e governança corporativa) foram abordados em $77 \%$ dos artigos selecionados, demonstrando uma predominância de interesse dos pesquisadores por estes tópicos. Essa realidade está em consonância com Paiva, Oliveira e Melo (2008) ao afirmarem que a "pulverização de esforços em termos temáticos" pode demonstrar falta de foco das pesquisas e levar ao enfraquecimento do 
direcionamento do campo de pesquisa.

O presente estudo também identificou que a maioria dos trabalhos desenvolvidos nos últimos anos (91\%) foram por meio de pesquisas com abordagem qualitativa no campo de empresas familiares, enquanto pesquisas quantitativas foram identificadas em apenas 9\% dos estudos selecionados. Tal prevalência sugere, então, uma oportunidade para pesquisas futuras com a aplicação de técnicas quantitativas que podem trazer contribuições para o avanço e crescimento do interesse acadêmico pelo campo de estudos sobre empresas familiares.

Por fim, verificou-se que as técnicas de coleta de dados mais empregadas nas pesquisas sobre empresas familiares têm sido entrevistas semiestruturadas e pesquisas documentais em bases de dados, enquanto técnicas como entrevistas em profundidade, survey, observação não participante e observação participante foram identificadas, juntas, em apenas $20 \%$ dos artigos selecionados. Tal preponderância também deixa em aberto uma janela para que pesquisadores da área possam empregar diferentes técnicas de coletas de dados, como incidente critico, grupos focais, pesquisa-ação, entre outros métodos.

A revisão da literatura é indispensável obter uma ideia precisa sobre o estado atual dos conhecimentos sobre um dado tema, as suas lacunas e a contribuição da investigação para o desenvolvimento do conhecimento. Portanto, espera-se que o presente estudo contribua para a construção de novos estudos relacionados ao campo de pesquisa sobre empresas familiares, já que este é um assunto ainda bastante inexplorado no contexto acadêmico brasileiro. Além disso, acredita-se que as percepções levantadas aqui poderão ser futuramente expandidas para outras áreas da pesquisa em administração, como empreendedorismo, cultura organizacional, entre outras áreas para que haja uma compreensão geral sobre o posicionamento do campo de estudos em administração nos últimos anos.

\section{REFERÊNCIAS}

Almeida-Santos, P. S.; Dani, A. C.; Magro, C. B. D.; Machado, D. G. (2017). Propriedade familiar e o earnings management no Brasil: uma análise sob a ótica do take a bath e do income smoothing. Enfoque Reflexão Contábil, Maringá, v. 36.

Barbosa, E. F. (2008). Instrumento de coleta de dados em pesquisas educacionais. Disponível em: <http://www.serprofessoruniversitario.pro.br>. Acesso em: setembro/2019.

Bertucci, J. L. O.; Silva, E. A.; Pimentel, T. D.; Pereira, R. D. (2009). Mecanismos de governança e processos de sucessão: um estudo sobre a influência dos elementos da governança corporativa na orientação do processo sucessório em uma empresa familiar. Revista Brasileira de Gestão de Negócios, v. 11, n. 31, p. 152-167.

Borges, A. F. et al. (2016). Empreendedorismo em Empresas Familiares: A pesquisa atual e os desafios futuros. RAM, Rev. Adm. Mackenzie, São Paulo, v. 17, n. 2, p. 93-121.

Botelho, L. L. R.; Cunha, C. C. A.; Macedo, M. (2011). O método da revisão integrativa nos estudos organizacionais. Gestão e Sociedade, v. 5, n. 11, p. 121- 36.

Cançado, V. L.; Lima, J. B.; Muylder, C. F.; Castanheira, R. B. (2013). Ciclo de vida, sucessão e processo de governança em uma empresa familiar: um estudo de caso no Grupo Seculus. Rev. Eletrônica de Administração. Porto Alegre, v. 19, n. 2, p. 485-516. 
Carvalho, C. C.; et al. (2016). Mensuração da Capacidade de Governança Corporativa das Empresas Familiares para migração ao Novo Mercado da BM\&FBovespa. Revista de Administração FACES Journal, v. 15, n. 3, p. 124-142.

Cavedon, R. N. (2008). Antropologia para Administradores. 2. ed. - Porto Alegre: Editora da UFRGS.

Costa, L. S. P.; et al. (2015). De Pai para Filho: A Sucessão em Pequenas e Médias Empresas Familiares. Contabilidade, Gestão e Governança, v. 18, n. 1, p. 61-82.

Ferreira, L. B.; Torrecilha, N.; Machado, S. H. S. (2012). A técnica de observação em estudos de administração. XXXVI Encontro da ANPAD, Rio de Janeiro. Anais do Encontro Nacional da Anpad.

Freitas, E. C.; Barth, M. (2012). De pai para filho: a complexidade e os desafios da gestão das empresas familiares. Revista de Administração da UFSM, v. 5, n. 3, p. 549-568.

Fonseca, E.; Parra, P.; Azevedo, M.; Lopes, P. (2010). A secretária executiva no processo de governança corporativa na empresa familiar. Revista de Gestão e Secretariado, v. 1, n. 1, p. 1-24.

Gerhardt, T. E.; Silveira, D. T. (2009). Métodos de pesquisa. Porto Alegre: Ed. da UFRGS.

Gil, A. C. (2006). Métodos e técnicas de pesquisa social. 5. ed. São Paulo: Atlas.

Gil, A. C. (2009). Como elaborar projetos de pesquisa. 4. ed. São Paulo: Atlas.

Goncalves, J.; Sérgio R. C. (2000). As empresas familiares no Brasil. Rev. adm. empresas, São Paulo, v. 40, n. 1, p. 7-12.

Grzybovski, D.; Vieira, L. (2012). Ciclo de vida das empresas familiares brasileiras de pequeno porte: uma análise das características e dinâmica estrutural em uma rede de revenda de gás liquefeito de petróleo. Teoria e Prática em Administração, v. 2, n. 2, p. 7896.

Lakatos, E. M; Marconi, M. A. (2009). Técnicas de pesquisa: planejamento e execução de pesquisas, amostragens e técnicas de pesquisa, elaboração, análise e interpretação de dados. 7. ed. São Paulo: Atlas.

Leone, M. (2005). Sucessão na empresa familiar: preparando as mudanças para garantir sobrevivência no mercado globalizado. São Paula: Atlas.

Lescura, C.; Brito, M. J.; Borges, A. F.; Cappelle, M. (2012). Representações Sociais sobre as Relações de Parentesco: Estudo de Caso em um Grupo Empresarial Familiar. Revista de Administração Contemporânea, v. 16, n. 1, p. 98-117.

Lourenço, C. D. S.; Ferreira, P. A. (2012). Cultura organizacional e mito fundador: um estudo de caso em uma empresa familiar. Gestão \& Regionalidade, v. 28, n. 84, p. 61-76.

Martins, A.; Maccari, E. A.; Campanário, M. A.; Almeida, M. I. R. (2008). Empresa familiar e as dificuldades enfrentadas pelos membros da terceira geração. Revista de Ciências da Administração, v. 10, n. 22, p. 30-54.

Miles, M. B. (1979). Qualitative data as an attractive nuisance: the problem of analysis. Administrative Science Quarterly.

Moraes Filho, A. C. T.; Barone, F. M.; Pinto, M. O. (2011). A produção científica em empresas familiares: um enfoque conceitual. Revista de Administração Pública, v. 45, n. 
6, p. 1971-1991.

Moré, C. L. (2015). A "entrevista em profundidade" ou "semiestruturada", no contexto da saúde. Investigação Qualitativa em Ciências Sociais - CIAIQ.

Oliveira, J. L.; Albuquerque, A. L.; Pereira, R. D. (2012). Governança, Sucessão e Profissionalização em uma Empresa Familiar: (re)arranjando o lugar da família multigeracional. Revista Brasileira de Gestão de Negócios, v. 14, n. 43, p. 176-192.

Padilla-Melendez, A.; Dieguez-Soto, J.; Garrido-Moreno, A. (2015). Pesquisa empírica sobre Inovação na Empresa Familiar: revisão de literatura e proposta de estrutura integrativa. Rev. Brasileira de Gestão e Negócios; São Paulo, v. 17, n. 56, p. 1064-1089.

Paiva, K.; Oliveira, M.; Melo, M. (2008). Produção científica brasileira sobre empresa familiar - um metaestudo de artigos publicados em anais de eventos da ANPAD no período de 1997-2007. RAM, Rev. Adm. Mackenzie, São Paulo, v. 9, n. 6, p. 148-173.

Paranhos, R.; et al. (2013). Corra que o survey vem aí: noções básicas para cientistas sociais. Revista Latinoamericana de Metodología de la Investigación Social, Buenos Aires, n. 6, p. 7-24.

Pereira, A.; Vieira, A.; Garcia, F.; Roscoe, M. (2013). Desconstrução do mito e sucessão do fundador em empresas familiares. Rev. adm. contemp., Curitiba, v. 17, n. 5, p. 518-535.

PWC. Pesquisa Global de Empresas Familiares. (2016). Disponível em <http:/www. pwc.com.br/pesquisa-global-empresas-familiares-2016>. Acesso em 12 de agosto de 2019.

Schneider, C.; Neto, L.M. (2006). Avaliação do desempenho organizacional da empresa familiar indústrias Alfa S.A. com base no modelo de gestão estratégica balanced scorecard. Revista de Gestão- USP, Vol.13, no 1, pp. 89-104.

Sharma, P., Chrisman, J.; Chua, J. (1997). Strategic management of the Family business: past research and future challenges. Family Business Review, 10, 1-35.

Teston, S. F.; Filippim, E. S. (2016). Perspectivas e Desafios da Preparação de Sucessores para Empresas Familiares. Revista de Administração Contemporânea, v. 20, n. 5, p. 524545.

Tres, D. L.; et al. (2014). Ciclo de vida organizacional: um estudo de caso em uma empresa familiar. Anais SEMEAD.

Triviños, A. N. S. (1987). Introdução à pesquisa em ciências sociais: a pesquisa qualitativa em educação. São Paulo: Atlas. 175p. 